\title{
An efficient algorithm for a generalized joint replenishment problem
}

\author{
J.B.G. Frenk* M.J. Kleijn* $\quad$ R. Dekker*
}

February 14, 1997

\begin{abstract}
In most multi-item inventory systems, the ordering costs consist of a major cost and a minor cost for each item included. Applying for every individual item a cyclic inventory policy, where the cycle length is a multiple of some basic cycle time, reduces the major ordering costs. An efficient algorithm to determine the optimal policy of this type is discussed in this paper. It is shown that this algorithm can be used for deterministic multi-item inventory problems, with general cost rate functions and possibly service level constraints, of which the well-known joint replenishment problem is a special case. Some useful results in determining the optimal control parameters are derived, and worked out for piecewise linear cost rate functions. Numerical results for this case show that the algorithm significantly outperforms other solution methods, both in the quality of the solution as in the running time.
\end{abstract}

Keywords: Inventory, Multi-item, Joint replenishment problem, Deterministic demand.

\section{Introduction}

Although in most of the literature on inventory theory single-item models are analyzed, in practice one often needs to determine stocking policies for multiple items. In most multi-item inventory systems, the ordering costs consist of a major ordering cost $c>0$ and a minor ordering $\operatorname{cost} c_{i}>0$ if item $i, 1 \leq i \leq n$, is ordered (Brown [3], Goyal [7, 8, 9], Goyal \& Satir [11], Kaspi \& Rosenblatt [16], Naddor [17], Silver [20]). For such a cost structure, coordination of replenishments will save major ordering costs and, as will be shown, this can be done efficiently if the inventory level for each item $i$ is controlled by a cyclic rule. The associated stocking policy $\left(R, k_{1}, \ldots, k_{n}\right) \in \mathbb{R}_{+} \times \mathbb{N}^{n}$ is to place a replenishment order every $R$ time units, and to include item $i$ in one out of every $k_{i}$ replenishments, $1 \leq i \leq n$. In the literature the average cost of such a policy is evaluated in two ways. The first takes account of so-called empty replenishment occasions which occur when the smallest frequency

\footnotetext{
*Erasmus University Rotterdam, Econometric Institute, P.O. Box 1738, 3000 DR Rotterdam, The Netherlands
} 
$k_{i}, 1 \leq i \leq n$, is larger than one. This induces for the major ordering costs a complicated correction factor $\Delta(\boldsymbol{k}), \boldsymbol{k}=\left(k_{1}, \ldots, k_{n}\right)$, equal to

$$
\Delta(\boldsymbol{k})=\sum_{i=1}^{n}(-1)^{i+1} \sum_{\{\alpha \subset\{1, \ldots, n\}:|\alpha|=i\}}\left(\operatorname{lcm}\left(k_{\alpha_{1}}, \ldots, k_{\alpha_{i}}\right)\right)^{-1}
$$

with $\operatorname{lcm}\left(k_{\alpha_{1}}, \ldots, k_{\alpha_{i}}\right)$ denoting the least common multiple of the integers $k_{\alpha_{1}}, \ldots, k_{\alpha_{i}}$ (Dagpunar [4]). With this correction factor the average cost of the above stocking policy is given by

$$
\Delta(\boldsymbol{k}) c / R+\sum_{i=1}^{n} \Phi_{i}\left(k_{i} R\right)
$$

where $\Phi_{i}\left(k_{i} R\right)$ denotes the minimum average cost of item $i$ replenished every $k_{i} R$ time units. However, in most papers (see the overviews of Goyal \& Satir [11] and Kaspi \& Rosenblatt [16]) this correction factor is set equal to one, and so the average cost of the stocking policy $\left(R, k_{1}, \ldots, k_{n}\right)$ reduces to

$$
c / R+\sum_{i=1}^{n} \Phi_{i}\left(k_{i} R\right)
$$

If the value $\varphi_{i}\left(k_{i} R\right)$ denotes the minimum holding and shortage cost of item $i$ during a replenishment cycle of length $k_{i} R$ time units, then it follows that

$$
\Phi_{i}\left(k_{i} R\right)=\frac{c_{i}+\varphi_{i}\left(k_{i} R\right)}{k_{i} R}
$$

The associated optimal stocking policy is now given by an optimal solution of either the optimization problem with correction factor

$$
\inf \left\{\Delta(k) c / R+\sum_{i=1}^{n} \Phi_{i}\left(k_{i} R\right): R>0, k_{i} \in I N, i=1, \ldots, n\right\}
$$

or the optimization problem without correction factor

$$
\inf \left\{c / R+\sum_{i=1}^{n} \Phi_{i}\left(k_{i} R\right): R>0, k_{i} \in I N, i=1, \ldots, n\right\}
$$

Due to the complicated correction factor $\Delta(\boldsymbol{k})$ the optimization problem $\left(Q_{c}\right)$ is much more difficult to solve than the optimization problem $(Q)$. Recently, Dekker, Frenk \& Wildeman [5] have developed an efficient algorithm, which is presented in Section 2, to solve the optimization problem $(Q)$ if the functions $\varphi_{i}$ and $\Phi_{i}, 1 \leq i \leq n$, satisfy the following property.

Property 1.1 For every $1 \leq i \leq n$ the function $\varphi_{i}:(0, \infty) \rightarrow I R$ is convex and the optimization problem $\inf \left\{\Phi_{i}(x): 0<x<\infty\right\}$ has a finite optimal solution $x_{i}^{*}>0$.

Moreover, after the execution of the algorithm we also obtain an indication of the quality of the generated solution for $(Q)$ with respect to the optimization problem $\left(Q_{c}\right)$. This algorithm was originally developed for maintenance models where the value $\varphi_{i}\left(k_{i} R\right)$ can be seen as the expected cost due to failures if component $i$ is maintained every $k_{i} R$ time units. In a subsequent paper, Wildeman, Frenk \& Dekker [24] specialized their method to the joint 
replenishment problem with the inventory level of every item controlled by the economic order quantity model. In this model, it is assumed that the demand process is deterministic with demand rate $\lambda_{i}>0$ for item $i, i=1, \ldots, n$, the lead time of any replenishment order is equal to zero and no shortages are allowed. The last condition (for every item) implies that the long-run fraction of demand satisfied directly from stock on hand equals one and so this condition can be seen as a service level constraint. Moreover, the value $h_{i}>0$ denotes the unit inventory holding cost of item $i$ per unit of time, and the corresponding cost rate function $f_{i}: \mathbb{R} \rightarrow[0,+\infty)$ is given by

$$
f_{i}(x)= \begin{cases}h_{i} x & \text { if } x \geq 0 \\ 0 & \text { otherwise }\end{cases}
$$

Observe, for general cost rate functions $f_{i}: \mathbb{R} \rightarrow[0, \infty)$, the inventory holding and shortage costs during the interval $[t, t+\Delta t)$ with $\Delta t$ small is approximated by $f_{i}\left(x_{t}\right) \Delta t$ if $x_{t}$ denotes the (positive or negative) inventory level of item $i$ at time $t$. It is well-known that for the economic order quantity model the function $\varphi_{i}$ is given by $\varphi_{i}(R)=\frac{1}{2} h_{i} \lambda_{i} R^{2}$ and this function is clearly convex on $(0, \infty)$. Moreover, the function $\Phi_{i}$ is given by $\Phi_{i}(R)=c_{i} / R+\frac{1}{2} h_{i} \lambda_{i} R$ and this function has a finite minimum attained at $R^{*}=\left(2 c_{i} / \lambda_{i} h_{i}\right)^{1 / 2}$.

In Section 3 it is shown that the method of Dekker, Frenk \& Wildeman [5] can be applied to deterministic multi-item inventory models with or without a service level constraint and general cost rate functions. Although the analysis can easily be extended for non-negative deterministic replenishment lead times, we assume that the lead time of any replenishment order equals zero. If a service level constraint is included, this replaces the shortage costs and so for both models (with or without a service level constraint) we associate a class of cost rate functions. For cost rate functions corresponding to a deterministic inventory model without a service level constraint we impose the following natural condition.

Property 1.2 For every $i \in I \subseteq\{1, \ldots, n\}$ the function $f_{i}: \mathbb{R} \rightarrow[0, \infty)$ is strictly positive and decreasing on $(-\infty, 0)$ and strictly increasing on $(0, \infty)$. Moreover, the function $f_{i}$ is continuous on $\mathbb{R} \backslash\{0\}$ and satisfies $f_{i}\left(0^{+}\right):=\lim _{x \downarrow 0} f_{i}(x)=0$.

Examples of such cost rate functions are the convex, concave or quadratic holding and shortage cost functions, discussed by Porteus [18] in his overview on stochastic inventory models. Naddor [17] analyzes the case of expensive storage of items, where the cost of holding $x$ units is given by $\alpha x^{m}$ with $\alpha>0$ and $m>1$ fixed. Arrow, Karlin \& Scarf [1] consider a convex penalty cost function for the case where small shortages are of little consequence, but large ones create more than proportionally great difficulties for the customers. Inventory models with both fixed and time-dependent shortage costs, which are discussed among others by Hadley \& Whitin [13] and Federgruen, Groenevelt \& Tijms [6], can be represented by cost rate functions satisfying Property 1.2. A special case of such a model is the lost-sales model with unit holding $\operatorname{cost} h_{i}$, fixed $\operatorname{cost} \pi_{i}$ per stockout and demand rate $\lambda_{i}$, which can be represented by the cost rate function

$$
f_{i}(x)= \begin{cases}h_{i} x & \text { if } x \geq 0 \\ \lambda_{i} \pi_{i} & \text { otherwise }\end{cases}
$$


At the end of Section 3, we will consider in detail cost rate functions with linear shortage costs and piecewise linear holding costs. The latter situation may arise when storage of products requires the use of several sources of limited capacity with different unit costs. Observe if these sources are used in order of ascending unit cost, the holding cost function will be convex (see also Veinott [23]). Non-convex piecewise linear holding costs may be the result of using sources of different capacities. Finally, a piecewise linear cost rate function can be used to approximate a general cost rate function.

For cost rate functions corresponding to a deterministic inventory model with a service level constraint, the next natural condition is imposed.

Property 1.3 For every $i \in\{1, \ldots, n\} \backslash$ I the function $f_{i}: \mathbb{R} \rightarrow[0, \infty)$ is continuous, strictly increasing on $[0, \infty)$ and vanishes on $(-\infty, 0)$.

Observe that the cost rate functions $f_{i}$ associated with the economic order quantity model, given by (1), satisfy Property 1.3 .

In the next section the algorithm of Dekker, Frenk \& Wildeman [5] will be presented. In Section 3 it is shown under which conditions on the cost rate functions Property 1.1 is satisfied. Moreover, results which are useful in deriving the optimal control parameters for each item $i$ are derived. Section 3 is concluded by presenting results for a special class of cost rate functions, i.e. piecewise linear holding costs and linear shortage costs. The results of our numerical experiments are presented in Section 4. It will be shown that the algorithm significantly outperforms well-known iterative solution procedures, both in the quality of the solution as in the running time. Finally, in Section 5 the main conclusions are given.

\section{An efficient algorithm for multi-item inventory problems}

In this section we summarize the algorithm which was developed by Dekker, Frenk \& Wildeman [5] to determine optimal cyclic maintenance frequencies in multi-component systems. The optimization problem $(Q)$, discussed in the introduction, can be solved efficiently by this algorithm if Property 1.1 is satisfied. In Section 3 it will be shown that for both types of models discussed in Section 1, with cost rate functions satisfying Property 1.2 or Property 1.3, the function $\varphi_{i}$ is convex on $(0, \infty)$. Hence, to apply the algorithm, we only need to verify that the function $\Phi_{i}$ has a finite minimum for every $i$. As will be proved, a sufficient condition is given by $\lim _{|x| \rightarrow \infty} f_{i}(x)=\infty, i \in I$, and $\lim _{x \rightarrow \infty} f_{i}(x)=\infty$ for $i \in\{1, \ldots, n\} \backslash I$. For more detailed information on the algorithm the reader is referred to [5]. Before discussing the algorithm we first transform the problems $(Q)$ and $\left(Q_{c}\right)$ by means of the transformation $R \rightarrow 1 / T$ into the equivalent problems

$$
\inf \left\{c T+\sum_{i=1}^{n} \Phi_{i}\left(k_{i} / T\right): T>0, k_{i} \in I N, i=1, \ldots, n\right\}
$$

and

$$
\inf \left\{\Delta(\boldsymbol{k}) c T+\sum_{i=1}^{n} \Phi_{i}\left(k_{i} / T\right): T>0, k_{i} \in I N, i=1, \ldots, n\right\}
$$


If $v(\cdot)$ denotes the optimal objective value of the optimization problem $(\cdot)$ then clearly $v(Q)$ equals $v(P)$ and $v\left(Q_{c}\right)$ equals $v\left(P_{c}\right)$. Moreover, a feasible solution $\left(T(P), k_{1}(P), \ldots, k_{n}(P)\right)$ of $(P)$ is optimal if and only if $\left(1 / T(P), k_{1}(P), \ldots, k_{n}(P)\right)$ is an optimal solution of $(Q)$. Since the objective function of $(P)$ is separable in $k_{i}, i=1, \ldots, n$, it follows that $(P)$ reduces to

$$
\inf \left\{c T+\sum_{i=1}^{n} \inf \left\{\Phi_{i}\left(k_{i} / T\right): k_{i} \in N\right\}: T>0\right\}
$$

By relaxing the constraints $k_{i} \in I N, i=1, \ldots, n$ by $k_{i} \geq 1$ we now consider the relaxation

$$
\inf \left\{c T+\sum_{i=1}^{n} \inf \left\{\Phi_{i}\left(k_{i} / T\right): k_{i} \geq 1\right\}: T>0\right\}
$$

It can be shown (Lemma 3.1 of [5]) without any conditions on the functions $\varphi_{i}(\cdot), i=1, \ldots, n$, that $v\left(P_{R}\right) \leq v\left(P_{c}\right) \leq v(P)$. If for $i=1, \ldots, n$ the function $g_{i}:(0, \infty) \rightarrow \mathbb{R}$ is given by

$$
g_{i}(T):=\inf \left\{\Phi_{i}\left(k_{i} / T\right): k_{i} \geq 1\right\}
$$

then by Property 1.1 we obtain that

$$
g_{i}(T)= \begin{cases}\Phi_{i}(1 / T) & \text { if } T \leq 1 / x_{i}^{*} \\ \Phi_{i}\left(x_{i}^{*}\right) & \text { otherwise }\end{cases}
$$

with $x_{i}^{*}:=\arg \min \left\{\Phi_{i}(x): x>0\right\}$ (which by Property 1.1 exists and is finite). Moreover, it follows that $g_{i}$ is convex and so the optimization problem $\left(P_{R}\right)$ given by

$$
\inf \left\{c T+\sum_{i=1}^{n} g_{i}(T): T>0\right\}
$$

is a one-dimensional convex programming problem. Since any optimal solution $T\left(P_{R}\right)$ of $\left(P_{R}\right)$ satisfies $0<T\left(P_{R}\right) \leq \max \left\{1 / x_{i}^{*}: i=1, \ldots, n\right\}$ (Lemma 3.2 of [5]) we may use the bisection algorithm, or golden-section search (see Chapter 8 of Bazaraa, Sherali \& Shetty [2]) to locate an optimal solution $T\left(P_{R}\right)$ of $\left(P_{R}\right)$. Under certain conditions (see Lemma 4.3 of [5]) one can show that the feasible solution $\left(T\left(P_{R}\right), 1, \ldots, 1\right)$ is an optimal solution for $(P)$ and so, if these easy verifiable conditions are satisfied, the problem is solved. If not, we determine an optimal solution of the optimization problem $(F P)$ given by $\inf \left\{\Phi_{i}\left(k_{i} / T\left(P_{R}\right)\right): k_{i} \in \mathbb{N}\right\}, i=1, \ldots, n$, and this generates a feasible solution $\left(T\left(P_{R}\right), k_{1}(F P), \ldots, k_{n}(F P)\right)$ of $(P)$ with

$$
k_{i}(F P):=\arg \min \left\{\Phi_{i}\left(k_{i} / T\left(P_{R}\right)\right): k_{i} \in I\right\}
$$

Observe by Property 1.1 that the optimization problem ( $F P$ ) can be solved easily (cf. [5]) and the next feasibility procedure yields exactly its optimal solution for $i=1, \ldots, n$.

\section{Feasibility Procedure}

For each $i=1, \ldots, n$ apply the following steps:

1. Compute $k=\left\lfloor T\left(P_{R}\right) x_{i}^{*}\right\rfloor$ with $\lfloor\cdot\rfloor$ the lower-entier function. 
2. If $k=0$ then $k_{i}(F P)=1$

3. If $k \geq 1$ then $k_{i}(F P)=k$ or $k_{i}(F P)=k+1$ depending on whether $\Phi_{i}\left(k / T\left(P_{R}\right)\right) \leq$ $\Phi_{i}\left((k+1) / T\left(P_{R}\right)\right)$ or $\Phi_{i}\left(k / T\left(P_{R}\right)\right) \geq \Phi_{i}\left((k+1) / T\left(P_{R}\right)\right)$

If the value $v(F P)$ is given by

$$
v(F P):=c T\left(P_{R}\right)+\sum_{i=1}^{n} \Phi_{i}\left(k_{i}(F P) / T\left(P_{R}\right)\right)
$$

then by our previous observations we obtain

$$
v(F P) \geq v(P) \geq v\left(P_{c}\right) \geq v\left(P_{R}\right)
$$

Hence, if $v(F P)$ is close to $v\left(P_{R}\right)$ we know that the objective value of the feasible solution $\left(T\left(P_{R}\right), k_{1}(F P), \ldots, k_{n}(F P)\right)$ is close to the optimal objective value of $(P)$ and $\left(P_{c}\right)$. If, given a tolerance, it is not close enough, we try to improve the feasible solution by the following procedure.

\section{Improved-Feasibility Procedure}

1. Let $k_{i}(I F P)=k_{i}(F P), i=1, \ldots, n$, with $k_{i}(F P)$ the values given by the above feasibility procedure.

2. Solve the optimization problem

$$
\min \left\{c T+\sum_{i=1}^{n} \Phi_{i}\left(k_{i}(I F P) / T\right): T>0\right\}
$$

and let $T(I F P)$ be an optimal value for $T$.

3. Determine new constants $k_{i}(I F P)$ by applying the feasibility procedure to the value $T(I F P)$, and let $v(I F P)$ be the corresponding objective value.

This procedure can be repeated with in step 1 the constants $k_{i}(F P)$ replaced by $k_{i}(I F P)$, and this can be done until no further improvement is found. It can be shown ([5]) that $v(I F P) \leq v(F P)$ and so every time the improved feasibility procedure is used, the corresponding solution is at least as good as the previous one. Observe by Property 1.1 that the optimization problem in step 2 of the improved-feasibility procedure is a one-dimensional convex optimization problem. If the final objective value $v(I F P)$ is still not close to $v\left(P_{R}\right)$, then a univariate Lipschitz optimization algorithm (see Horst \& Pardalos [15]) can be applied. Before applying such an algorithm, a lower and an upper bound on the optimal value $T(P)$ of $(P)$ must be determined. Since $\left(P_{R}\right)$ is a convex-programming problem, an upper bound $T_{\text {up }}$ is given by the smallest $T \geq T\left(P_{R}\right)$ for which the objective function of $\left(P_{R}\right)$ equals $v(I F P)$ (Lemma 4.6 of [5]). If there also exists a value $T_{\text {low }}$ given by the largest $T \leq T\left(P_{R}\right)$ for which the objective function of $\left(P_{R}\right)$ equals $v(I F P)$, then this value is a lower bound on $T(P)$ (Lemma 4.7 of $[5])$. Otherwise, we take the lower bound $T_{\text {low }}=(1 / c)\left(v(I F P)-\sum_{i=1}^{n} \Phi_{i}\left(x_{i}^{*}\right)\right)$ 
(Lemma 4.5 of [5]). Finally, a Lipschitz constant of the objective function of $(P)$ on the interval $\left[T_{\text {low }}, T_{\text {up }}\right]$ must be determined. For this the reader is referred to Appendix A of [5]. Due to the special structure of the objective function of $(P)$ it can be shown that the Lipschitz constant of this objective function is decreasing in $T$ on $\left[T, T_{u p}\right]$ and so we may use an improved version of Evtushenko's algorithm (cf.[5]). We now summarize the results by presenting the following solution procedure for $(Q)$ :

1. Solve the convex-programming problem $\left(P_{R}\right)$ and use $0<T\left(P_{R}\right) \leq \max _{1 \leq i \leq n} 1 / x_{i}^{*}$. An optimal value $T\left(P_{R}\right)$ can be found by applying a bisection algorithm if the objective function of $\left(P_{R}\right)$ is differentiable, or otherwise by applying golden-section search.

2. If $T\left(P_{R}\right) \leq \min _{1 \leq i \leq n} 1 / x_{i}^{*}$ then the vector $\left(T\left(P_{R}\right), 1, \ldots, 1\right)$ is optimal for $(P)$ and $\left(P_{c}\right)$; stop.

3. If $T\left(P_{R}\right)>\min _{1 \leq i \leq n} 1 / x_{i}^{*}$, check whether the objective function of $\left(P_{R}\right)$ evaluated in $\min _{1 \leq i \leq n} 1 / x_{i}^{*}$ equals $v\left(P_{R}\right)$. If so, the vector $\left(T\left(P_{R}\right), 1, \cdots, 1\right)$ is optimal for $(P)$ and $\left(P_{c}\right) ;$ stop.

4. Otherwise, we first find a feasible solution for $(P)$ by applying the feasibility procedure or the improved-feasibility procedure. If the corresponding objective value is within a certain tolerance of $v\left(P_{R}\right)$, then this also applies to $v(P)$; stop.

5. If this does not happen and therefore the solution is not good enough, apply a globaloptimization technique on the interval $\left[T_{l o w}, T_{u p}\right]$ to find a value for $T(P)$.

To conclude this section, we consider a policy introduced by Goyal \& Soni [12], who allow for multiple cycle times. In their paper they consider three basic cycle times, i.e. $T_{1}=T$, $T_{2}=3 T$ and $T_{3}=5 T$, which implies that $k_{i}, i=1, \ldots, n$, can attain values from the set $\{1,1.5,2,2.5,3,4,4.5,5,6,7,7.5, \ldots\}$. It is easy to see that the optimal objective value $v(G S)$ of this problem must satisfy $v\left(P_{R}\right) \leq v(G S) \leq v(P)$, and thus our method also provides information a bout the optimal costs of the more general class of coordination policies suggested by Goyal \& Soni [12].

In the next section we will consider a general class of deterministic multi-item inventory problems to which the above solution procedure can efficiently be applied.

\section{Analysis of the underlying models}

In this section we consider a simple deterministic multi-item inventory model, where all demand is backlogged, and the inventory for each item $i, 1 \leq i \leq n$, is controlled by a periodic review, order-up-to level policy. If for any $1 \leq i \leq n$ the function $f_{i}$ satisfies Property 1.2 (corresponding to an inventory model without a service level constraint) or Property 1.3 (corresponding to an inventory model with a service level constraint) then for this model the function $\varphi_{i}$, introduced in Section 1, will be derived and shown to be convex. This implies, if the functions $\Phi_{i}, i=1, \ldots, n$, have a finite minimum on $(0, \infty)$, that Property 1.1 is satisfied and so the method of Dekker, Frenk \& Wildeman [5] can be applied. A sufficient and weak 
condition to guarantee that $\Phi_{i}$ has a finite minimum will also be discussed in this section. Since we first verify the convexity property of the underlying inventory model of an arbitrary item $i$, the subscript $i$ will henceforth be omitted. In the so-called $(R, S)$-policy, every $R>0$ time units a replenishment order is placed with $\operatorname{cost} c$, to raise the inventory position to the order-up-to level $S$, with $-\infty<S<\infty$. Although the results can easily be extended for non-negative deterministic replenishment lead times, we assume that the lead time equals zero. The demand rate is constant and deterministic, and denoted by $\lambda>0$. We start our analysis by considering an inventory model without a service level constraint. The analysis of an inventory model with a service level constraint is much easier and will be carried out afterwards.

\section{Models without a service level constraint}

To determine for the inventory model without a service level constraint the function $\varphi(\cdot)$, we observe that the holding and shortage costs for an arbitrary $(R, S)$ policy during a cycle of length $R$ are given by

$$
I(R, S):=\int_{0}^{R} f(S-\lambda t) d t
$$

and the average costs for an arbitrary $(R, S)$ policy equal

$$
g(R, S):=\frac{c+I(R, S)}{R}
$$

Hence, the minimum holding and shortage costs during a cycle of length $R$ are

$$
\varphi(R):=\inf \{I(R, S):-\infty<S<\infty\}
$$

and so

$$
\Phi(R):=\inf \{g(R, S):-\infty<S<\infty\}=\frac{c+\varphi(R)}{R}
$$

We first consider the optimization problem associated with the objective function $\varphi(R)$. Since by Property 1.2 the function $f$ is decreasing and continuous on $(-\infty, 0)$ it follows for every $S_{1}<S_{2} \leq 0$ that

$$
I\left(R, S_{1}\right)=\int_{0}^{R} f\left(S_{1}-\lambda t\right) d t \geq \int_{0}^{R} f\left(S_{2}-\lambda t\right) d t=I\left(R, S_{2}\right)
$$

Moreover, since $f$ is strictly increasing and continuous on $(0, \infty)$ with $f\left(0^{+}\right)=0$, one can show similarly that $I\left(R, S_{1}\right)>I\left(R, S_{2}\right)$ for every $S_{1}>S_{2} \geq \lambda R$, and so the optimization problem associated with the optimal objective value $\varphi(R), R>0$, reduces to

$$
\varphi(R)=\inf \{I(R, S): 0 \leq S \leq \lambda R\}
$$

Since the function $S \rightarrow I(R, S)$ is continuous and the feasible set of $\left(P_{\varphi(R)}\right)$ is compact, we obtain that the set of optimal solutions of $\left(P_{\varphi(R)}\right)$ is nonempty and so

$$
\varphi(R)=\min \{I(R, S): 0 \leq S \leq \lambda R\}
$$


Before characterizing in Theorem 3.1 an optimal solution of $\left(P_{\varphi(R)}\right)$, we introduce for every function $f$ satisfying Property 1.2 the value

$$
R_{f}^{*}:=\sup \left\{R \geq 0: f(\lambda R)-f\left(0^{-}\right) \leq 0\right\}
$$

with $f\left(0^{-}\right):=\lim _{x \uparrow 0} f(x)$. Since $f$ is continuous and strictly increasing on $(0, \infty)$ it follows that $R_{f}^{*}$ is the unique solution of the equation $f(\lambda R)-f\left(0^{-}\right)=0$. This implies that $R_{f}^{*}=0$ if and only if $f$ is continuous in 0 .

Theorem 3.1 If Property 1.2 holds, the optimization problem $\left(P_{\varphi(R)}\right), R>0$, has a unique optimal solution denoted by $S(R)$, and it satisfies $S(R)=\lambda R$ if $0<R \leq R_{f}^{*}$ and $0<S(R)<$ $\lambda R$ if $R>R_{f}^{*}$. Moreover, for $R>R_{f}^{*}$ the optimal solution $S(R)$ is the unique solution on $(0, \lambda R)$ of the equation $f(S)-f(S-\lambda R)=0$.

Proof: Since the function $f$ might only have a discontinuity at zero, it follows for every $R>0$ that the partial derivative $\frac{\partial I}{\partial S}(R, S)$ of the function $I(R, S)=\int_{0}^{R} f(S-\lambda t) d t$ exists for every $0<S<\lambda R$ and on $(0, \lambda R)$ this partial derivative is given by

$$
\frac{\partial I}{\partial S}(R, S)=\frac{1}{\lambda}(f(S)-f(S-\lambda R))
$$

Since the function $f$ satisfies Property 1.2 it follows for every $0<R \leq R_{f}^{*}$ and $0<S<\lambda R$ that

$$
f(S)-f(S-\lambda R)<f(\lambda R)-f\left(0^{-}\right) \leq f\left(\lambda R_{f}^{*}\right)-f\left(0^{-}\right) \leq 0
$$

and so $\frac{\partial I}{\partial S}(R, S)<0$ for every $0<S<\lambda R \leq \lambda R_{f}^{*}$. This implies by the continuity of the function $S \rightarrow I(R, S)$ that $\lambda R$ is the unique optimal solution of $\left(P_{\varphi(R)}\right)$ for every $0<R \leq R_{f}^{*}$. Moreover, since Property 1.2 implies

$$
\frac{\partial I}{\partial S}\left(R, 0^{+}\right):=\lim _{S \downarrow 0} \frac{\partial I}{\partial S}(R, S)=\frac{1}{\lambda}\left(f\left(0^{+}\right)-f(-\lambda R)\right)=\frac{-f(-\lambda R)}{\lambda}<0
$$

and the function $S \rightarrow \frac{\partial I}{\partial S}(R, S)$ is continuous and strictly increasing on $(0, \lambda R)$, there exists for every $R>R_{f}^{*}$ a unique value $0<S(R)<\lambda R$ satisfying $\frac{\partial I}{\partial S}(R, S(R))=0$ and this proves the desired result.

The next result discusses some important properties of the function $R \rightarrow S(R)$.

Lemma 3.1 If Property 1.2 holds then the function $R \rightarrow S(R)$ is continuous and increasing on $(0, \infty)$.

Proof: We only give a proof of the above result for $R_{f}^{*}>0$ since the proof for $R_{f}^{*}=0$ can easily be adapted. To start the proof, we first show that the function $R \rightarrow S(R)$ is increasing on $(0, \infty)$. By Theorem 3.1 it is sufficient to verify this property on $\left[R_{f}^{*}, \infty\right)$. Consider therefore $R_{1}>R_{2} \geq R_{f}^{*}>0$. Since by Property 1.2 the function $f$ is decreasing on $(-\infty, 0)$ it follows for every $0<S<\lambda R_{2}<\lambda R_{1}$ that $f\left(S-\lambda R_{1}\right) \geq f\left(S-\lambda R_{2}\right)$ and hence

$$
f(S)-f\left(S-\lambda R_{1}\right) \leq f(S)-f\left(S-\lambda R_{2}\right)
$$


for every $0<S<\lambda R_{2}$. If we assume by contradiction that $S\left(R_{1}\right)<S\left(R_{2}\right)$ it follows by Theorem 3.1 that $S\left(R_{1}\right)<\lambda R_{2}$ and this yields by (4) that

$$
f\left(S\left(R_{1}\right)\right)-f\left(S\left(R_{1}\right)-\lambda R_{1}\right) \leq f\left(S\left(R_{1}\right)\right)-f\left(S\left(R_{1}\right)-\lambda R_{2}\right)
$$

Since the function $S \rightarrow f(S)-f\left(S-\lambda R_{2}\right)$ is strictly increasing on $\left(0, \lambda R_{2}\right)$ this implies for $R_{2}>R_{f}^{*}$ that $S\left(R_{1}\right)<S\left(R_{2}\right)<\lambda R_{2}$ and hence by Theorem 3.1 we obtain the contradiction

$$
\begin{aligned}
0 & =f\left(S\left(R_{1}\right)\right)-f\left(S\left(R_{1}\right)-\lambda R_{1}\right) \leq f\left(S\left(R_{1}\right)\right)-f\left(S\left(R_{1}\right)-\lambda R_{2}\right) \\
& <f\left(S\left(R_{2}\right)\right)-f\left(S\left(R_{2}\right)-\lambda R_{2}\right)=0
\end{aligned}
$$

For $R_{1}>R_{2}=R_{f}^{*}$ it follows by a similar argument that

$$
\begin{aligned}
0 & =f\left(S\left(R_{1}\right)\right)-f\left(S\left(R_{1}\right)-\lambda R_{1}\right) \leq f\left(S\left(R_{1}\right)\right)-f\left(S\left(R_{1}\right)-\lambda R_{2}\right) \\
& <f\left(\lambda R_{f}^{*}\right)-f\left(0^{-}\right)=0
\end{aligned}
$$

and again we have derived a contradiction. Hence, the function $R \rightarrow S(R)$ is increasing and to verify the continuity of this function we first observe by the monotonicity that $b_{+}\left(R_{f}^{*}\right):=$ $\lim _{R \downarrow R_{f}^{*}} S(R)$ exists and satisfies $S\left(R_{f}^{*}\right) \leq b_{+}\left(R_{f}^{*}\right)$. Moreover, for every $R>R_{f}^{*}$ we know by Theorem 3.1 that $S(R)<\lambda R$ and this implies $b_{+}\left(R_{f}^{*}\right) \leq \lambda R_{f}^{*}$. Since $S\left(R_{f}^{*}\right)=\lambda R_{f}^{*}$ (Theorem 3.1) it follows that $b_{+}\left(R_{f}^{*}\right)=S\left(R_{f}^{*}\right)$ and so $R \rightarrow S(R)$ is continuous at $R=R_{f}^{*}$. Also, for $R_{1}>R_{f}^{*}$ we obtain that $b_{+}\left(R_{1}\right):=\lim _{R \downarrow R_{1}} S(R)$ exists. By Theorem 3.1 and $R \rightarrow S(R)$ increasing it follows that $R \rightarrow S(R)-\lambda R$ is decreasing and this shows

$$
b_{+}\left(R_{1}\right)-\lambda R_{1}=\lim _{R \downarrow R_{1}}(S(R)-\lambda R) \leq S\left(R_{1}\right)-\lambda R_{1}<0
$$

and

$$
b_{+}\left(R_{1}\right)=\lim _{R \downarrow R_{1}} S(R) \geq S\left(R_{1}\right)>0
$$

Applying now the continuity of $f$ on $\mathbb{R} \backslash\{0\}$ yields

$$
0=\lim _{R \downarrow R_{1}} f(S(R))-f(S(R)-\lambda R)=f\left(b_{+}\left(R_{1}\right)\right)-f\left(b_{+}\left(R_{1}\right)-\lambda R_{1}\right)
$$

and again by Theorem 3.1 it follows that $b_{+}\left(R_{1}\right)=S\left(R_{1}\right)$. Hence, we have shown that the function $R \rightarrow S(R)$ is right-continuous in $R=R_{1}$. Similarly, it can be shown that $b_{-}\left(R_{1}\right):=\lim _{R \uparrow R_{1}} S(R)=S\left(R_{1}\right)$ and so $R \rightarrow S(R)$ is left-continuous in $R=R_{1}$, implying the desired result.

To verify that the function $\varphi(\cdot)$ is convex on $(0, \infty)$ we first need to prove the following result.

Lemma 3.2 If $\mathcal{K}$ denotes the convex cone given by

$$
\mathcal{K}=\{(R, S): R>0,0 \leq S \leq \lambda R\}
$$

and Property 1.2 holds, then the function $(R, S) \rightarrow I(R, S)$ is convex on $\mathcal{K}$. 
Proof: For

$$
F(x):= \begin{cases}\int_{0}^{x} f(z) d z & \text { if } x \geq 0 \\ -\int_{x}^{0} f(z) d z & \text { otherwise }\end{cases}
$$

it follows by Property 1.2 that the function $x \rightarrow F(x)$ is strictly convex on $[0, \infty)$ and concave on $(-\infty, 0)$. Observe for every $(R, S) \in \mathbb{R}_{+} \times \mathbb{R}$ that

$$
I(R, S)=\frac{1}{\lambda}(F(S)-F(S-\lambda R))
$$

Hence, for every two distinct points $\left(R_{i}, S_{i}\right) \in \mathcal{K}, i=1,2$, and $0<\alpha<1$ we obtain that

$$
\begin{aligned}
& I\left(\alpha R_{1}+(1-\alpha) R_{2}, \alpha S_{1}+(1-\alpha) S_{2}\right)= \\
& \frac{1}{\lambda}\left(F\left(\alpha S_{1}+(1-\alpha) S_{2}\right)-F\left(\alpha\left(S_{1}-\lambda R_{1}\right)+(1-\alpha)\left(S_{2}-\lambda R_{2}\right)\right)\right)
\end{aligned}
$$

and this implies by the strict convexity of $F$ on $[0, \infty)$ and the concavity on $(-\infty, 0)$ that

$$
\begin{aligned}
& I\left(\alpha R_{1}+(1-\alpha) R_{2}, \alpha S_{1}+(1-\alpha) S_{2}\right) \\
& \leq \frac{1}{\lambda}\left(\alpha F\left(S_{1}\right)+(1-\alpha) F\left(S_{2}\right)-\alpha F\left(S_{1}-\lambda R_{1}\right)-(1-\alpha) F\left(S_{2}-\lambda R_{2}\right)\right) \\
& =\alpha I\left(R_{1}, S_{1}\right)+(1-\alpha) I\left(R_{2}, S_{2}\right)
\end{aligned}
$$

which shows the desired result.

The convexity of the function $\varphi$ is now an immediate consequence of Lemma 3.2, as will be shown in the next theorem.

Theorem 3.2 If Property 1.2 holds then the function $\varphi$ is convex on $(0, \infty)$.

Proof: Let $R_{1}, R_{2} \in \mathbb{R}_{+}$and $0<\alpha<1$ be given. By Theorem 3.1 it follows that $\alpha S\left(R_{1}\right)+(1-\alpha) S\left(R_{2}\right) \leq \lambda\left(\alpha R_{1}+(1-\alpha) R_{2}\right)$. Applying now Lemma 3.2 and the definition of $\varphi(R)$, we obtain

$$
\begin{aligned}
\varphi\left(\alpha R_{1}+(1-\alpha) R_{2}\right) & \leq I\left(\alpha R_{1}+(1-\alpha) R_{2}, \alpha S\left(R_{1}\right)+(1-\alpha) S\left(R_{2}\right)\right) \\
& \leq \alpha I\left(R_{1}, S\left(R_{1}\right)\right)+(1-\alpha) I\left(R_{2}, S\left(R_{2}\right)\right) \\
& =\alpha \varphi\left(R_{1}\right)+(1-\alpha) \varphi\left(R_{2}\right)
\end{aligned}
$$

which proves the desired result.

In Theorem 3.2 it is shown that the function $\varphi$ is convex on $(0, \infty)$ and this implies by Theorem 11B of Roberts \& Varberg [19] that for every $R>0$ the right-derivative

$$
\varphi_{+}^{\prime}(R):=\lim _{h \downarrow 0} \frac{\varphi(R+h)-\varphi(R)}{h}
$$

and the left-derivative

$$
\varphi_{-}^{\prime}(R):=\lim _{h \downarrow 0} \frac{\varphi(R-h)-\varphi(R)}{-h}
$$

exist. By definition, the function $\varphi$ is differentiable on $(0, \infty)$ if $\varphi_{+}^{\prime}(R)=\varphi_{-}^{\prime}(R)$ for every $R>0$. A slightly stronger result is now proved in the following theorem. 
Theorem 3.3 If Property 1.2 holds then the function $\varphi$ is continuously differentiable on $(0, \infty)$. Moreover, the derivative $\varphi^{\prime}(R)$ of $\varphi$ at the point $R$ is given by $f(S(R))$.

Proof: We only show the result for $R_{f}^{*}>0$ since the proof for $R_{f}^{*}=0$ is similar. If $0<R \leq R_{f}^{*}$ it follows by Theorem 3.1 that

$$
\varphi(R)=\int_{0}^{R} f(\lambda R-\lambda t) d t=\frac{1}{\lambda} \int_{0}^{\lambda R} f(x) d x
$$

This implies by the continuity of the function $f$ on $(0, \infty)$ that $\varphi_{+}^{\prime}(R)=f(\lambda R)=f(S(R))$ for every $0<R<R_{f}^{*}$ and $\varphi_{-}^{\prime}(R)=f(\lambda R)=f(S(R))$ for every $0<R \leq R_{f}^{*}$. Hence, the function $\varphi$ is differentiable on $\left(0, R_{f}^{*}\right)$ and its left-derivative $\varphi_{-}^{\prime}\left(R_{f}^{*}\right)$ is given by $f\left(\lambda R_{f}^{*}\right)=f\left(S\left(R_{f}^{*}\right)\right)$. Consider now some $R \geq R_{f}^{*}$. By the definition of $\varphi$ it follows for every $h>0$ that

$$
\begin{aligned}
\varphi(R+h)-\varphi(R) & \leq \int_{0}^{R+h} f(S(R)-\lambda t) d t-\int_{0}^{R} f(S(R)-\lambda t) d t \\
& =\int_{R}^{R+h} f(S(R)-\lambda t) d t
\end{aligned}
$$

Since by Theorem 3.1 we know that $S(R)<\lambda R$ this implies by the continuity of the function $f$ on $(0, \infty)$ that $\varphi_{+}^{\prime}(R) \leq f(S(R)-\lambda R)$ for every $R>R_{f}^{*}$. Similarly, we obtain for $R-h>R_{f}^{*}$ with $h>0$ that

$$
\varphi(R-h)-\varphi(R) \leq-\int_{R-h}^{R} f(S(R)-\lambda t) d t
$$

and as before it follows that $\varphi_{-}^{\prime}(R) \geq f\left(S(R)-\lambda R\right.$ ) for every $R>R_{f}^{*}$. Since the function $\varphi$ is convex we know that $\varphi_{-}^{\prime}(R) \leq \varphi_{+}^{\prime}(R)$ for every $R>0$ (Roberts \& Varberg [19]) and so the inequality

$$
f(S(R)-\lambda R) \leq \varphi_{-}^{\prime}(R) \leq \varphi_{+}^{\prime}(R) \leq f(S(R)-\lambda R)
$$

holds for every $R>R_{f}^{*}$. By Theorem 3.1 it follows that $f(S(R)-\lambda R)=f(S(R)$ ) for every $R>R_{f}^{*}$ and this implies by the above inequality that the function $\varphi$ is differentiable on $\left(R_{f}^{*}, \infty\right)$ with $\varphi^{\prime}(R)=f(S(R))$. From $(7)$ it is easy to verify that $\varphi_{+}^{\prime}\left(R_{f}^{*}\right) \leq f\left(0^{-}\right)=$ $f\left(\lambda R_{f}^{*}\right)=f\left(S\left(R_{f}^{*}\right)\right)$ and since $\varphi_{-}^{\prime}\left(R_{f}^{*}\right)=f\left(S\left(R_{f}^{*}\right)\right)$ we obtain by the convexity of $\varphi$ in a similar way as before that $\varphi^{\prime}\left(R_{f}^{*}\right)$ equals $f\left(S\left(R_{f}^{*}\right)\right)$. Hence, we have shown that $\varphi^{\prime}(R)=f(S(R))$ for every $R>0$, and by Lemma 3.1 and the continuity of $f$ on $(0, \infty)$ we finally obtain that the function $\varphi$ is continuously differentiable on $(0, \infty)$.

As will be shown at the end of this section, the above result is extremely useful in deriving analytical formulas for $\varphi$ if $f$ has some special form. Moreover, it can also be used to compute the Lipschitz constant for our global optimization procedure discussed in Section 2.

\section{Models with a service level constraint}

We will now consider a deterministic inventory model with a service level constraint and so we assume that the cost rate function satisfies Property 1.3. Let $\beta(R, S)$ denote the long-run 
fraction of demand for some item satisfied directly from stock on hand if an $(R, S)$-policy is used. By the regenerative structure of the inventory position process it follows that

$$
\beta(R, S)=\frac{\text { total demand satisfied directly from stock in one cycle }}{\text { total demand in one cycle }}
$$

Since the denominator equals $\lambda R$ we obtain that

$$
\beta(R, S)= \begin{cases}0 & \text { if } S<0 \\ S / \lambda R & \text { if } 0 \leq S \leq \lambda R \\ 1 & \text { otherwise }\end{cases}
$$

As before, the holding costs during one cycle are given by

$$
I(R, S)=\int_{0}^{R} f(S-\lambda t) d t
$$

and the average costs for an arbitrary $(R, S)$-policy equal

$$
g(R, S):=\frac{c+I(R, S)}{R}
$$

The minimum holding costs during a cycle of fixed length $R$, subject to the service level constraint $\beta(R, S) \geq \beta$, with $0<\beta \leq 1$ fixed, are now given by

$$
\varphi(R):=\inf \{I(R, S):-\infty<S<\infty, \beta(R, S) \geq \beta\}
$$

and thus

$$
\Phi(R):=\inf \{g(R, S):-\infty<S<\infty, \beta(R, S) \geq \beta\}=\frac{c+\varphi(R)}{R}
$$

Since by Property 1.3 the cost rate function $f$ is increasing on $\mathbb{R}$ and vanishes on $(-\infty, 0)$, the optimization problem associated with $\varphi(R)$ is trivial to solve and by $(8)$ we obtain that

$$
\begin{aligned}
\varphi(R) & =\inf \left\{\int_{0}^{R} f(S-\lambda t) d t: S \geq \beta \lambda R\right\} \\
& =\int_{0}^{R} f(\beta \lambda R-\lambda t) d t \\
& =\int_{0}^{\beta R} f(\beta \lambda R-\lambda t) d t
\end{aligned}
$$

implying that $S(R)=\beta \lambda R$ for $R>0$. Hence, it follows by a similar argument as in Lemma 3.2 that $\varphi$ is convex on $(0, \infty)$. Moreover, it is also easy to verify by the continuity of $f$ that

$$
\varphi^{\prime}(R)=\beta f(\beta \lambda R)
$$

and so we have completed the analysis of a deterministic inventory model with a service level constraint. 


\section{Verification of Property 1.1}

The first part of Property 1.1 is satisfied, since for both models it was shown that the function $\varphi$ is convex and continuously differentiable. This implies by Theorem I.1.1.6 of Hiriart-Urruty \& Lemarechal [14] that the function $R \rightarrow \Phi(1 / R)$ also satisfies these properties. By this observation, the next result is easy to prove.

Theorem 3.4 The optimization problem inf $\{\Phi(x): 0<x<\infty\}$ has a finite optimal solution $x^{*}$ if and only if the finite value $x^{*}$ is a solution of the equation $\Phi^{\prime}(x)=0$.

The above result is useful to compute a finite optimal solution if one exists. A sufficient condition to guarantee that such a finite optimal solution exists is given by the next result.

Theorem 3.5 For any $f$ satisfying Property 1.2 the optimization problem in Theorem 3.4 has a finite optimal solution if $\lim _{|x| \rightarrow \infty} f(x)=\infty$. The same result holds for any $f$ satisfying Property 1.3 if $\lim _{x \rightarrow \infty} f(x)=\infty$.

Proof: We only prove the first part, since the proof of the second part is almost identical. From (3), (5), (6) and $c>0$ it follows that

$$
g(R, S)>\frac{1}{\lambda R}(F(S)-F(S-\lambda R))
$$

This implies by the nonnegativity of the function $f$ that for every $(R, S) \in \mathcal{K}$ (defined in Lemma 3.2) and $S \leq \frac{1}{2} \lambda R$ we have

$$
g(R, S)>\frac{-1}{\lambda R} F(S-\lambda R) \geq \frac{-1}{\lambda R} F\left(-\frac{1}{2} \lambda R\right)
$$

Similarly, for every $(R, S) \in \mathcal{K}$ and $S \geq \frac{1}{2} \lambda R$ it follows that

$$
g(R, S)>\frac{1}{\lambda R} F(S) \geq \frac{1}{\lambda R} F\left(\frac{1}{2} \lambda R\right)
$$

and so we obtain that

$$
g(R, S)>\frac{1}{\lambda R} \min \left\{F\left(\frac{1}{2} \lambda R\right),-F\left(-\frac{1}{2} \lambda R\right)\right\}
$$

for every $(R, S) \in \mathcal{K}$. Since $\lim _{|x| \rightarrow \infty} f(x)=\infty$ it follows that $\lim _{R \rightarrow \infty} F\left(\frac{1}{2} \lambda R\right) / \lambda R=\infty$ and $\lim _{R \rightarrow \infty}-F\left(-\frac{1}{2} \lambda R\right) / \lambda R=\infty$ and this shows that the function $\Phi$ has a finite positive minimum point.

For most inventory models the above condition on the cost rate function is very natural, and in some sense also necessary. To show this, we consider again the lost-sales model, for which the cost rate functions are given by (2). In this case the property $\lim _{|x| \rightarrow \infty} f_{i}(x)=\infty$ is not satisfied. By Theorem 3.1 we obtain that

$$
S(R)= \begin{cases}\lambda R & \text { if } R \leq R_{f}^{*} \\ \lambda R_{f}^{*} & \text { otherwise }\end{cases}
$$


where $R_{f}^{*}=\pi / h$, and thus the function $\varphi(R)$ is given by

$$
\varphi(R)= \begin{cases}\frac{1}{2} \lambda h R^{2} & \text { if } R \leq R_{f}^{*} \\ \lambda \pi R-\frac{1}{2} \lambda h R_{f}^{*} & \text { otherwise }\end{cases}
$$

Observe that $\varphi(R)$ is continuously differentiable and convex on $(0, \infty)$. Using straightforward calculations, one can verify that $\Phi^{\prime}(R)<0$ on $(0, \infty)$ if $(\lambda \pi)^{2}<2 c \lambda h$, and so the optimization problem $\inf \{\Phi(x): 0<x<\infty\}$ does not have a finite optimal solution in this case, implying that the method of Dekker, Frenk \& Wildeman [5] can in general not be applied for the lost-sales model. We conclude this section by considering a special case of a cost rate function $f$ satisfying Property 1.2 .

\section{A piecewise linear cost rate function}

Assume that the cost rate function $f$ is given by

$$
f(x)= \begin{cases}-p x & x<0 \\ h_{1} x & 0 \leq x<a_{1} \\ \vdots & \vdots \\ f\left(a_{i-1}\right)+h_{i}\left(x-a_{i-1}\right) & a_{i-1} \leq x<a_{i}, \quad i=2, \ldots, m-1 \\ \vdots & \vdots \\ f\left(a_{m-1}\right)+h_{m}\left(x-a_{m-1}\right) & a_{m-1} \leq x<\infty\end{cases}
$$

where $p>0$ and $h_{i}>0$ for all $i$. Hence, we have piecewise linear holding costs and linear shortage costs. In Figure 1 this cost rate function is illustrated. Observe that for general cost rate functions $f$, numerical integration procedures are needed to evaluate $\varphi(R)$ and $\Phi(R)$. If we use the trapezoidal rule (see Stummel \& Hainer [21]), the cost rate function is replaced by a piecewise linear function. Therefore, the analysis below will provide a useful tool for analyzing more general cost rate functions.

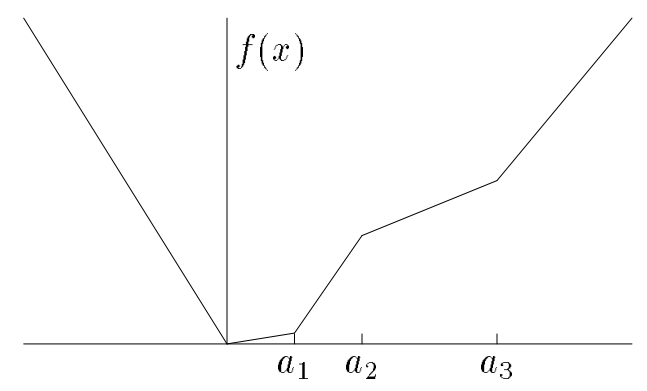

Figure 1: A piecewise linear cost rate function.

Since $f$ is continuous in 0 it follows immediately that $R_{f}^{*}=0$. For $i=1, \ldots, m-1$ we define $R_{i}$ such that $S\left(R_{i}\right)=a_{i}$. By Theorem 3.1 it follows that this corresponds to

$$
f\left(a_{i}\right)-f\left(a_{i}-\lambda R_{i}\right)=0 \quad \Rightarrow \quad R_{i}=\left(f\left(a_{i}\right)+p a_{i}\right) / \lambda p
$$


Moreover, we define $R_{0}:=0, a_{0}:=0, R_{m}:=\infty$ and $a_{m}:=\infty$. The next lemma determines for a given $R>0$ the value of $S(R)$.

Lemma 3.3 For $R_{i-1} \leq R<R_{i}, i=1, \ldots, m$, it follows that

$$
a_{i-1} \leq S(R)<a_{i}
$$

In particular,

$$
S(R)=a_{i-1}+\frac{p}{p+h_{i}} \lambda\left(R-R_{i-1}\right)
$$

Proof: Since $S\left(R_{i-1}\right)=a_{i-1}$ and $S\left(R_{i}\right)=a_{i}$ the first part immediately follows by Lemma 3.1 . To prove the second part we observe that

$$
\begin{aligned}
f(S(R))-f(S(R)-\lambda R) & =f\left(a_{i-1}\right)+h_{i}\left(S(R)-a_{i-1}\right)+p(S(R)-\lambda R) \\
& =\left(p+h_{i}\right) S(R)+f\left(a_{i-1}\right)-h_{i} a_{i-1}-p \lambda R
\end{aligned}
$$

By setting this expression equal to zero we obtain that

$$
\begin{aligned}
S(R) & =\frac{p}{p+h_{i}} \lambda R+\frac{h_{i} a_{i-1}-f\left(a_{i-1}\right)}{p+h_{i}} \\
& =\frac{p}{p+h_{i}} \lambda\left(R-R_{i-1}\right)+a_{i-1}
\end{aligned}
$$

which shows the desired result.

By Theorem 3.1 and Theorem 3.3 we obtain that the derivative of $\varphi(R)$ is given by

$$
\varphi^{\prime}(R)=f(S(R)-\lambda R)
$$

for $R>R_{f}^{*}=0$. This implies by Lemma 3.3 for $R_{i-1} \leq R<R_{i}, i=1, \ldots, m$ that

$$
\varphi^{\prime}(R)=-p\left(a_{i-1}-\lambda R_{i-1}-\frac{h_{i}}{p+h_{i}} \lambda\left(R-R_{i-1}\right)\right)
$$

and also

$$
\varphi^{\prime \prime}(R)=\lambda h_{i} \frac{p}{p+h_{i}}
$$

Hence, by Theorem 3.2 it follows that $\varphi(\cdot)$ is a convex, piecewise quadratic function, and so

$$
\varphi(R)=\varphi\left(R_{i-1}\right)+p\left(\lambda R_{i-1}-a_{i-1}\right)\left(R-R_{i-1}\right)+\frac{1}{2} \lambda h_{i} \frac{p}{p+h_{i}}\left(R-R_{i-1}\right)^{2}
$$

The derivative of $\Phi(R)$ for $R>0$ is given by

$$
\Phi^{\prime}(R)=\frac{R \varphi^{\prime}(R)-(c+\varphi(R))}{R^{2}}
$$

and thus by the above observations we obtain for $R_{i-1} \leq R<R_{i}$ that

$$
\Phi^{\prime}(R)=\frac{1}{R^{2}}\left[p\left(\lambda R_{i-1}-a_{i-1}\right) R_{i-1}-c-\varphi\left(R_{i-1}\right)+\frac{1}{2} h_{i} \frac{p}{p+h_{i}} \lambda\left(R^{2}-R_{i-1}^{2}\right)\right]
$$

The next result is a direct application of Theorem 3.4 and Theorem 3.5. 
Lemma 3.4 Let $k:=\inf \left\{i=1, \ldots, m: \Phi^{\prime}\left(R_{i}\right)>0\right\}$. Then it follows that $R^{*}:=\arg \min \{\Phi(R):$ $R>0\}$ is given by

$$
R^{*}=\sqrt{\frac{2\left[c+\varphi\left(R_{k-1}\right)-p\left(\lambda R_{k-1}-a_{k-1}\right) R_{k-1}\right]}{\lambda h_{k} p /\left(p+h_{k}\right)}+R_{k-1}^{2}}
$$

Proof: By the definition of $k$ and Theorem 3.5, the value $R^{*}$ must be contained in the interval $\left[R_{k-1}, R_{k}\right)$. Using (10) and the necessary and sufficient first order optimality condition $\Phi^{\prime}\left(R^{*}\right)=0$ (see Theorem 3.4 ), the desired result follows.

Observe that (11) is equivalent with

$$
R^{*}=\alpha_{k} R_{k-1}
$$

for $k>1$, where

$$
\alpha_{k}:=\sqrt{1-2 \frac{\left(p+h_{k}\right)}{h_{k} p \lambda} \Phi^{\prime}\left(R_{k-1}\right)}
$$

This section is concluded by summarizing the above results in the following algorithm which efficiently determines the optimal replenishment interval $R^{*}$ if the cost rate function is piecewise linear.

Step 0 Let $c, \lambda, p, h_{1}, \ldots, h_{m}>0$ and $0<a_{1}<\cdots<a_{m-1}<\infty$ be given, and set $i:=0, a_{i}:=0, R_{i}=0, f\left(a_{i}\right):=0, \varphi\left(R_{i}\right):=0$ and $\Phi^{\prime}\left(R_{i}\right)=-\infty$

Step 1 While $\Phi^{\prime}\left(R_{i}\right) \leq 0$ and $i<m$ do:

$$
\begin{aligned}
& i:=i+1 \\
& f\left(a_{i}\right):=f\left(a_{i-1}\right)+h_{i}\left(a_{i}-a_{i-1}\right) \\
& R_{i}=\left(f\left(a_{i}\right)+p a_{i}\right) / \lambda p \\
& \varphi\left(R_{i}\right)=\varphi\left(R_{i-1}\right)+p\left(\lambda R_{i-1}-a_{i-1}\right)\left(R_{i}-R_{i-1}\right)+\frac{1}{2} \lambda h_{i} \frac{p}{p+h_{i}}\left(R_{i}-R_{i-1}\right)^{2} \\
& \Phi^{\prime}\left(R_{i}\right)=\left[p\left(\lambda R_{i}-a_{i}\right) R_{i}-c-\varphi\left(R_{i}\right)\right] / R_{i}^{2}
\end{aligned}
$$

Step $2 k:=i$ and $R^{*}$ is determined by (11)

Algorithm 3.1: An algorithm to calculate $R^{*}$ for a piecewise linear cost rate function.

\section{Numerical results}

In this section we will investigate the solution procedure for $(Q)$, described in Section 2, and compare it with the heuristic approaches of Goyal [7], Brown [3] and Goyal \& Gunasekaran [10]. Goyal [7] and Brown [3] apply an iterative algorithm, where they initialize each $k_{i}=1$ and then find the corresponding optimal $R$ by setting the derivative of the objective function of $(Q)$ equal to zero. Subsequently, they find for each $i$ a value of $k_{i}$, by setting the derivative of the objective function of $(Q)$ with respect to $k_{i}$ equal to zero, and rounding to the best integer value. Once a value for $k_{i}$ is found, it is compared to the $k_{i}$ in 
the previous iteration. When for each item $i$ the $k_{i}$ in two successive iterations are equal, the algorithm terminates. Otherwise, a new optimal $R$ is found for the current values of $k_{i}$ and the procedure is repeated. Goyal \& Gunesakaran [10] use the same procedure, but they obtain for each $i$ a value of $k_{i}$ by rounding the value of $k_{i}$, obtained by setting the derivative of the objective function of $(Q)$ equal to zero, to the nearest integer. This procedure is faster than the one of Goyal [7] and Brown [3], but will lead to poorer solutions. Although the above algorithms are fast, they cannot guarantee an optimal solution, and have the disadvantage that they are often stuck in a local optimal solution (see Van Egmond et al. [22]). The algorithm of Dekker, Frenk \& Wildeman [5] however guarantees an optimal solution, and it can also give in very little time a good approximation by solving the relaxed problem $\left(P_{R}\right)$ and applying a (improved) feasibility procedure. Using the objective value of the relaxed problem, it is possible to give an upper bound on the deviation of this approximated solution. This aspect is not shared by the other solution methods in the literature. Numerical experiments by Kaspi \& Rosenblatt [16] indicate that using the initial values of $k_{i}$ suggested by Silver [20] together with the iterative approach of Goyal [7] and Brown [3] leads to good results. However, to obtain the initial values of $k_{i}$ one needs to set the derivative of the objective function of $(Q)$ with respect to $R$ equal to zero, and substitute the corresponding value of $R$ into the objective function. The initial values of $k_{i}$ are then obtained by setting for $i=1, \ldots, n$ the derivative of the objective function with respect to $k_{i}$ equal to zero. This leads to solving a system of $n$ equations, which can be done analytically for the joint replenishment problem with no shortages allowed and cost rate functions given by (1) (see Silver [20]). However, for general cost rate functions numerical procedures are needed to obtain the initial values of $k_{i}$, and therefore this approach is not suitable for our situation.

In particular, we have tested the algorithm for the piecewise linear cost rate function which was discussed in Section 3. The algorithm was implemented in Borland Pascal 7.0 on a Compaq 486DX/66 personal computer. We selected 6 different values for the number of items $n$, and 7 different values for the major setup cost $c$. For the number of breakpoints $m$ in the holding cost function we analyzed 3 cases. This yields 126 different combinations of these parameters, and for each combination 25 problem instances were created by randomly choosing values for $h_{i k}, p_{i}, \lambda_{i}$ and $a_{i k}$. Hence, in total 3150 test problems were evaluated. In Table 1 the data are reported.

\begin{tabular}{lll}
\hline$n$ & $=$ & $3,5,7,10,25,50$ \\
$c$ & $=$ & $10,50,100,200,500,750,1000$ \\
$m$ & $=$ & $3,5,10$ \\
\hline$c_{i}$ & $\epsilon$ & {$[1,500]$} \\
$h_{i k}$ & $\epsilon$ & {$[1,20]$} \\
$p_{i}$ & $\epsilon$ & {$[5,500]$} \\
$\lambda_{i}$ & $\epsilon$ & {$[10,500]$} \\
$a_{i, k}-a_{i, k-1}$ & $\in$ & {$[1,50]$} \\
\hline
\end{tabular}

Table 1: Tested parameter values.

In Table 2 the relevant results of the algorithm for the 3150 test problems are presented. 
The deviations of the relaxation, the solutions obtained by the feasibility procedures and the solutions of the heuristics by Goyal [7], Brown [3] and Goyal \& Gunesakaran [10] were determined by comparing the objective values with the optimal objective value $v(P)$ obtained by Lipschitz optimization, where a maximum relative devation of $0.01 \%$ was allowed.

\begin{tabular}{lll}
\hline Relaxation $\left(P_{R}\right):$ & Average running time $(\mathrm{sec})$. & 0.02 \\
& Average deviation $\left(v(P)-v\left(P_{R}\right)\right) / v\left(P_{R}\right)$ & $0.599 \%$ \\
& Minimum deviation & $0.000 \%$ \\
& Maximum deviation & $2.273 \%$ \\
\hline Feasibility Procedure (FP): & Average running time (sec.) & 0.00 \\
& Average deviation $(v(F P)-v(P)) / v(P)$ & $0.276 \%$ \\
& Minimum deviation & $0.000 \%$ \\
& Maximum deviation & $3.984 \%$ \\
\hline Improved-Feasibility Procedure (IFP): & Average running time (sec.) & 0.21 \\
& Average deviation $(v(I F P)-v(P)) / v(P)$ & $0.090 \%$ \\
& Minimum deviation & $0.000 \%$ \\
& Maximum deviation & $3.003 \%$ \\
\hline Goyal and Brown (GES B): & Average running time $(\mathrm{sec})$. & 0.53 \\
& Average deviation $(v(G \& B)-v(P)) / v(P)$ & $0.705 \%$ \\
& Minimum deviation & $0.000 \%$ \\
& Maximum deviation & $11.880 \%$ \\
\hline Goyal \&; Gunesakaran $(G G):$ & Average running time $(\mathrm{sec})$. & 0.38 \\
& Average deviation $(v(G G)-v(P)) / v(P)$ & $1.205 \%$ \\
& Minimum deviation & $0.000 \%$ \\
& Maximum deviation & $17.300 \%$ \\
\hline
\end{tabular}

Table 2: Average results of 3150 test problems.

It can be seen from the table that solving the relaxation takes very little time (on average 0.02 seconds). Applying the feasibility procedure takes a negligible amount of time, and so we obtain within very little time a feasible solution for $(Q)$, with an average deviation of $0.276 \%$ and a maximum deviation of $3.984 \%$ in our test examples. Applying the improved-feasibility procedure also takes little time (on average 0.21 seconds), and leads to an average deviation from the optimal costs of $0.09 \%$ and a maximum deviation of $3.003 \%$. Hence, the algorithms of Goyal [7], Brown [3] and Goyal \& Gunesakaran [10] are outperformed, while the running times of the improved-feasibility procedure are smaller.

The deviations of $11.88 \%$ for the heuristic of Goyal [7] and Brown [3], and the deviation of $17.3 \%$ for the method of Goyal \& Gunesakaran [10] occur for one of the problem instances with $c=10$ and $n=7$. In Table 3 and Table 4 the parameters and results for these instances are given. Both heuristics are stuck in a local minimum after a small number of iterations, which is caused by the initialization of each $k_{i}$ at the value one. For both test problems the solutions obtained by solving the relaxation and applying the (improved) feasibility procedure were much better than the ones of Goyal [7] and Brown [3], and Goyal \& Gunesakaran [10] (compare $v(F P)$ and $v(I F P)$ with $v(G \& B))$. 


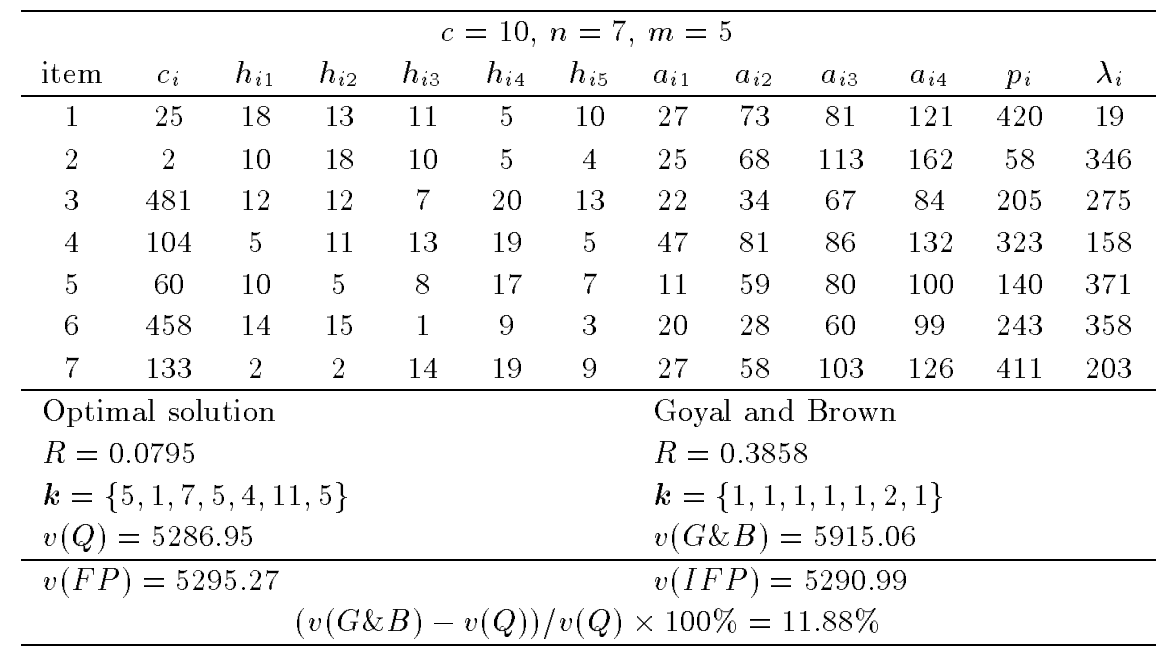

Table 3: Parameters and results of problem instance for which the heuristic of Goyal and Brown performs worst.

\begin{tabular}{ccccccccc}
\hline \multicolumn{10}{c}{$c=10, n=7, m=3$} \\
item & $c_{i}$ & $h_{i 1}$ & $h_{i 2}$ & $h_{i 3}$ & $a_{i 1}$ & $a_{i 2}$ & $p_{i}$ & $\lambda_{i}$ \\
\hline 1 & 490 & 1 & 1 & 1 & 30 & 63 & 74 & 405 \\
2 & 2 & 17 & 17 & 17 & 43 & 58 & 216 & 451 \\
3 & 63 & 8 & 16 & 6 & 3 & 24 & 412 & 78 \\
4 & 144 & 5 & 7 & 20 & 10 & 39 & 385 & 163 \\
5 & 171 & 5 & 14 & 9 & 12 & 48 & 430 & 148 \\
6 & 36 & 14 & 13 & 1 & 39 & 49 & 336 & 85 \\
7 & 32 & 15 & 1 & 11 & 8 & 47 & 459 & 15 \\
\hline Optimal solution & \multicolumn{7}{c}{$R=0.244$} \\
$R=0.059$ & $k=\{6,1,1,1,2,1,2\}$ \\
$k=\{26,1,6,6,8,4,9\}$ & $v(G G)=3705.89$ \\
$v(Q)=3159.33$ & \multicolumn{1}{c}{$v(I F P)=3159.37$} \\
\hline$v(F P)=3159.77$ & $(v(G G)-v(Q)) / v(Q) \times 100 \%=17.30 \%$ \\
\hline
\end{tabular}

Table 4: Parameters and results of problem instance for which the heuristic of Goyal \& Gunesakaran performs worst.

The running time of the Lipschitz optimization depends on the number of items and on the setup costs. The number of breakpoints $m$ did not have a significant influence on the running times. In Table 5 the average running times of the 75 examples in each class of test problems are reported. The running time appears to be increasing more than linearly in the number of items, which is caused by the fact that the interval $\left[T_{\text {low }}, T_{u p}\right]$ containing the optimal $T$ (see Section 2) tends to increase as $n$ increases. Since the objective function is steeper for larger values of $S$, which causes smaller upper bounds, the running times decrease as the value of $S$ increases. 


\begin{tabular}{ccccccc}
\hline & $n=3$ & $n=5$ & $n=7$ & $n=10$ & $n=25$ & $n=50$ \\
\hline$c=10$ & 0.24 & 0.64 & 1.09 & 2.13 & 9.44 & 21.42 \\
$c=50$ & 0.13 & 0.30 & 0.59 & 1.05 & 5.20 & 14.79 \\
$c=100$ & 0.09 & 0.26 & 0.47 & 0.88 & 4.26 & 11.89 \\
$c=200$ & 0.08 & 0.21 & 0.42 & 0.63 & 3.07 & 9.01 \\
$c=500$ & 0.08 & 0.14 & 0.25 & 0.44 & 2.36 & 6.74 \\
$c=750$ & 0.06 & 0.11 & 0.20 & 0.38 & 1.79 & 6.06 \\
$c=1000$ & 0.06 & 0.09 & 0.17 & 0.34 & 1.47 & 4.59 \\
\hline average & 0.11 & 0.25 & 0.46 & 0.83 & 3.94 & 18.64 \\
\hline
\end{tabular}

Table 5: Average running times (sec.) of the Lipschitz optimization.

\section{Conclusions}

In this paper we analyzed a generalized joint replenishment problem, with general cost rate functions and possibly service level constraints. It was shown that the algorithm of Dekker, Frenk \& Wildeman [5] can be used to solve this problem efficiently. The main advantage of this algorithm lies in the fact that it gives in very little time a good near-optimal solution with a known upper bound on the deviation, and, if necessary, it provides a guaranteed optimal solution using Lipschitz optimization. Useful results to determine the optimal control parameters were derived, and worked out for cost rate functions with piecewise linear holding costs and linear shortage costs. For this special case, the performance of the algorithm was compared with well-known heuristics, and it outperformed them both in the quality of the solution as in the running time.

Finally, we like to remark that although deterministic inventory models are not always realistic, they may be used to obtain a lower bound for stochastic inventory models. In a subsequent paper we will apply the procedure to stochastic inventory models with an $(R, S)$ inventory policy.

Acknowledgement: The authors thank Ralph Wildeman for writing the computer program.

\section{References}

[1] K.J. Arrow, S. Karlin, and H. Scarf. The nature and structure of inventory problems. In K.J. Arrow, S. Karlin, and H. Scarf, editors, Studies in the mathematical theory of inventory and production. Stanford University Press, Stanford, Ca., 1958.

[2] M.S. Bazaraa, H.D. Sherali, and C.N. Shetty. Nonlinear Programming: Theory and Algorithms. John Wiley \& Sons, New York, 1993.

[3] R.G. Brown. Decision Rules for Inventory Management. Holt, Reinhart and Winston, New York, 1967. 
[4] J.S. Dagpunar. Formulation of a multi item single supplier inventory problem. Journal of the Operational Research Society, 33:285-286, 1982.

[5] R. Dekker, J.B.G. Frenk, and R.E. Wildeman. How to determine maintenance frequencies for multi-component systems? a general approach. In S. Özekici, editor, Reliability and Maintenance of Complex Systems. Springer-Verlag, Berlin Heidelberg, 1996.

[6] A. Federgruen, H. Groenevelt, and H.C. Tijms. Coordinated replenishments in a multiitem inventory system with compound Poisson demands. Management Science, 30:344$357,1984$.

[7] S.K. Goyal. Determination of economic packaging frequency for items jointly replenished. Management Science, 20:232-235, 1973.

[8] S.K. Goyal. Determination of optimum packaging frequency of items jointly replenished. Management Science, 21:436-443, 1974.

[9] S.K. Goyal. A note on formulation of the multi-item single supplier inventory model. Journal of the Operational Research Society, 33:287-288, 1982.

[10] S.K. Goyal and A. Gunasekaran. Determining economic maintenance frequency of a transport fleet. International Journal of Systems Science, 4:655-659, 1992.

[11] S.K. Goyal and A.T. Satir. Joint replenishment inventory control: deterministic and stochastic models. European Journal of Operational Research, 38:2-13, 1989.

[12] S.K. Goyal and R. Soni. Economic packaging frequency of jointly replenished items with multiple manufacturing and packaging cycles. In A. Chikán, editor, Proceedings of the Third International Symposium on Inventories, pages 521-529. Akademiai Kioado, Budapest, 1986.

[13] G. Hadley and T.M. Whitin. Analysis of Inventory Systems. Prentice-Hall, Englewood Cliffs, N.J., 1963.

[14] J.B. Hiriart-Urruty and C. Lemarechal. Convex Analysis and Minimization Algorithms I. Springer-Verlag, Berlin, 1993.

[15] R. Horst and P.M. Pardalos. Handbook of Global Optimization. Kluwer Academic Publishers, Dordrecht, 1995.

[16] M. Kaspi and M.J. Rosenblatt. The effectiveness of heuristic algorithms for multi-item inventory systems with joint replenishment costs. International Journal of Production Research, 23:109-116, 1985.

[17] E. Naddor. Inventory Systems. John Wiley \& Sons, New York, 1966.

[18] E.L. Porteus. Stochastic inventory theory. In D.P. Heyman and M.J. Sobel, editors, Handbooks in OR \& MS, vol. 2. Elsevier Science Publishers B.V., North-Holland, 1990. 
[19] A.W. Roberts and D.E. Varberg. Convex Functions. Academic Press, New York, 1973.

[20] E.A. Silver. A simple method of determining order quantities in joint replenishments under deterministic demand. Management Science, 22:1351-1361, 1976.

[21] F. Stummel and K. Hainer. Introduction to Numerical Analysis. Scottish Academic Press, Edinburgh, 1980.

[22] R. Van Egmond, R. Dekker, and R.E. Wildeman. Correspondence on: Determining economic maintenance frequency of a transport fleet. International Journal of Systems Science, 26:1755-1757, 1995.

[23] A.F. Veinott Jr. The status of mathematical inventory theory. Management Science, $12: 745-777,1966$.

[24] R.E. Wildeman, J.B.G. Frenk, and R. Dekker. An efficient optimal solution method for the joint replenishment problem. to appear in the European Journal of Operational Research, 1996. 